\title{
Manejo médico de la osteoartrosis con glucosamina y condroitín sulfato
}

\section{Edgar J. Nieto}

Prof. Titular de Ortopedia y Traumatología. Universidad de Los Andes. Merida. Venezuela.

De las patologías inflamatorias articulares, la osteoartrosis u osteoartritis $(\mathrm{OA})$ es la forma más común, la principal causa de incapacidad en el adulto mayor, y a nivel de salud pública su impacto sigue en constante aumento y de manera especial en ancianos obesos. Hasta el día de hoy, no existe tratamiento definitivo para evitar su aparición o detener su progresión. Una mejor comprensión de la fisiopatología de la OA y a nivel clínico el desarrollo de biomarcadores más sensibles o estudios no invasivos, para identificar y poner a prueba los tratamientos, podrían reducir la carga de esta enfermedad común $n^{1,2}$.

En el anciano ocurren cambios en el cartílago y además en el músculo (sarcopenia), hueso (aumento de la remodelación y la pérdida de masa ósea), grasa (aumento de los depósitos), sistema nervioso (propiocepción alterada), etc; todos ellos, de manera independiente o en conjunto, juegan un papel en el desarrollo de la OA, el dolor y la pérdida de la normalidad de la función articular. Lo que aún falta es una conexión entre lo que sucede a nivel del condrocito envejecido y los cambios que ocurren en el resto de los tejidos. Teniendo en cuenta que la OA es impulsada por factores biomecánicos y acompañado de un bajo grado de inflamación crónica local, en todos los tejidos de la articulación, las terapias fallarán si ambos aspectos de la enfermedad no se consideran junto con los cambios sistémicos ${ }^{3}$.

La degeneración del cartílago articular no es de manera simple el resultado del envejecimiento y desgaste mecánico, porque a pesar de que la edad es el mayor factor de riesgo para la OA, pero no es una consecuencia inevitable del envejecer. Los mecanismos, para relacionarlos, se conocen por completo desde el punto de vista estadístico. Vale la pena destacar que el estrés celular y el daño oxidativo contribuyen a la inflamación crónica y que ambos promueven las enfermedades relacionadas con la edad. Los condrocitos envejecidos expresan el fenotipo secretor "senescentes", que tiene algunas características comunes con los de la OA, de manera especial, en la producción y la secreción de citoquinas proinflamatorias, como las qui- miocinas, y proteasas ${ }^{3,4}$.

Desde el punto de vista mecánico, para satisfacer las demandas, el hueso se adapta a las cargas al incrementar la remodelación, pero ellas generan alteraciones en el hueso subcondral, y es factible que jueguen un papel importante en el desarrollo de la OA. De manera especial en el recambio óseo y la mineralización, con la consecuente alteración del volumen y en la densidad aparente, que pueden afectar de forma negativa el medio ambiente biomecánico de la articulación. Modificaciones en el área debajo del cartílago, tales como lesiones de médula ósea y el desgaste óseo, tienden a ocurrir, de forma más frecuente en los compartimentos de la rodilla más cargadas, y están asociados con la pérdida de cartílago en la misma región ${ }^{5}$.

En lo referente al dolor, se ha asociado con cambios en la médula ósea, sinovitis y derrame. Se han encontrado receptores del dolor en la sinovia, ligamentos, cápsula y hueso subcondral, pero no en el cartílago. El hueso subcondral también se ha asociado con el dolor de rodilla, pero existe una pobre correlación entre la clínica y la radiología, es decir la relación entre osteofitos y dolor ha sido conflictiva. Si se llega a entender la secuencia y las consecuencias fisiopatológicas de la OA, se podrá tener un enfoque terapéutico racional. Es importante destacar, que los objetivos del tratamiento requieren la comprensión del papel de estas estructuras en el dolor, porque ésta es la razón por la cual los pacientes buscan atención médica ${ }^{6}$.

Una clara indicación de que los cambios en la $\mathrm{OA}$ se producen a nivel del hueso subcondral, serían los osteofitos, de aparición tardía y por esta razón la radiografía es relativamente insensible en la detección de los cambios tempranos. Con el advenimiento de las imágenes por resonancia magnética (RM), de la rodilla, se ha puesto de manifiesto que la mayoría de las consideradas "normales" en radiografías tienen alguna anormalidad detectable en la $\mathrm{RM}^{7}$.

Las estrategias de tratamiento para la OA incluyen las terapias no farmacológicas y con medicamentos. La pérdida de peso, no levantar peso y los ejercicios constituyen

\section{Correspondencia}

E-mail: Edgar.nieto@gmail.com 
las modalidades terapéuticas no farmacológicas. En lo que respecta a terapia con preparados, se dispone de analgésicos opiáceos o no, y los antiinflamatorios no esteroideos, ambos son opciones para la OA debido a su bien establecida eficacia.Sin embargo, el uso a largo plazo de estos fármacos puede inducir una serie de efectos secundarios, que llegan a ser graves, de manera especial en los ancianos. Por esta razón, la atención se ha centrado en la investigación y el desarrollo de nuevos tipos de medicamentos y tratamientos, que pueden mejorar los síntomas clínicos de la OA, con una mejor tolerabilidad y perfiles de seguridad que sean capaz de limitar el daño del cartílago y otros tejidos intraarticulares, como los fármacos sintomáticos de acción lenta para la OA, SYSADOAs, o los que tratan de modificarla, DMOADs ${ }^{8}$.

La idea de esta investigación es revisar el estado actual de las publicaciones relacionadas con el uso de la glucosamina y condroitín sulfato en el manejo médico de la OA, en vista de que su uso se ha incrementado de forma significativa, pero ha generado muchas controversias.

La glucosamina y el condroitín sulfato son miembros de un grupo de suplementos dietéticos a menudo denominados "agentes complementarios", o " drogas modificadoras de la enfermedad". Se derivan de cartílagos de animales o conchas de cangrejo y han sido promocionados para aliviar el dolor en la rodilla y tal vez la reparación de las células que recubren la articulación, revitalizando el cartílago dañado?.

La glucosamina es un amino monosacárido soluble en agua y precursor de la síntesis de proteínas glicosiladas y lípidos. Es uno de los monosacáridos más abundantes en el cuerpo y se encuentra en el mercado como un suplemento dietético, con una biodisponibilidad oral de aproximadamente el $20 \%{ }^{10}$.

Puede tener efectos anticatabólico y antiinflamatorio, en estudios experimentales; cuando se aplica a condrocitos y sinoviocitos inhibe mediadores, como la prostaglandina (PG)E2, óxido nítrico (NO), y metaloproteinasas de la matriz (MMPs). Inhibe la expresión de la ciclooxigenasa COX-2, la producción de prostaglandinas PGE2, el inductor de la sintasa del óxido nítrico iNOS, las metaloproteasas MMP-3, 2 y 9 y la síntesis de proteoglicanos (Akt). De igual manera con efecto sobre la síntesis y translocación al núcleo del factor nuclear kappa B (NF$\mathrm{kB}$ ), incluso en condrocitos humanos; por vía epigenética previene la expresión de IL-1 $\beta^{11-14}$.

Los estudios experimentales, en animales, han puesto en evidencia que actúa como inmunomodulador, inhibiendo la interleuquina $1 \beta$ (IL-1 $1 \beta$ ), la cual reduce la inflamación articular y la degradación del cartílago, y es- timula la síntesis de proteoglicanos. De manera similar, es un precursor y promotor de la síntesis de glicosaminoglicanos, o reductor de la degradación de este componente principal del cartílago. Pudiera contribuir a la síntesis de condroitín sulfato. Este efecto podría retrasar la progresión de la $\mathrm{OA}^{15,16}$.

En estudios in vitro, la adición de glucosamina a cultivos de condrocitos aumenta la síntesis de agrecanos y también puede ejercer un efecto antiinflamatorio, al reducir el efecto catabólico de la proteasa. De la misma manera, reducción de la metaloproteinasa-3 hace que la IL-1 estimule la producción de prostaglandinas y aumenta la tasa de síntesis de proteoglicano del cartílago articular ${ }^{14,17-18}$.

En estudios experimentales, en ratas, suprimió la sinovitis con mejoría de la alodinia mecánica. Por otra parte, la glucosamina atenuó la p38 y la c-Jun $\mathrm{N}$-terminal quinasa (JNK) y aumentó la expresión de la señal de la quinasa extracelular regulada 1/2 (ERK1/2) en el cartílago artrósico. De la misma manera se ha evidenciado elevación del producto de degradación del colágeno tipo II del cartílago (CTXII), y una supresión significativa de su determinación, luego de la administración de glucosamina ${ }^{19,20}$.

Desde el punto de vista histológico, en artrosis, se hace patente una reducción de la pérdida de proteoglicano, en el cartílago de la meseta tibial lateral pero no en el del cóndilo femoral. Del mismo modo, el análisis macroscópico de cartílago, mostró que la meseta tibial lateral solo tenía una tasa significativamente más baja de la enfermedad en el grupo de glucosamina, que fue consistente con los resultados de la evaluación histológica independiente. Se observó una reducción significativa en el contenido de gluco amino glicans GAG, en los cóndilos femorales de los tratados con placebo, pero no en la misma región de las tratadas con glucosamina. Este estudio no mostró cambios a nivel medial ${ }^{21}$.

Es conocido el papel del hueso subcondral en la producción de la OA y se ha demostrado que la glucosamina, en ratones, estimula la diferenciación de osteoblastos e inhibe la de los osteoclastos, asociado a la acción antiinflamatoria ${ }^{22}$.

Estudios relacionados con la mejoría clínica de la sintomatología, en animales, han revelado que disminuye, en el plasma, la concentración de prostaglandinas E2 y óxido nítrico; preserva el cartílago, incrementa los GAG, asociado a disminución de osteofitos y la erosión, de manera similar la sinovitis y la inflamación ósea entre otras ${ }^{23}$.

Para cualquier medicamento es de importancia determinar su concentración plasmática máxima, la cual ha sido determinada de manera correcta a la dosis de $1500 \mathrm{mg}$ diarios de sulfato de glucosamina, y a las 3 horas se alcanzan 
$10 \mu \mathrm{M}$ y se elimina en 15 horas. Uno de los errores del estudio GlcN hydrochloride (GlcN.H)/Chondroitin sulfate Arthritis Intervention Trial (GAIT), es que las concentraciones del cloruro de glucosamina son menores a las del sulfato. Todos los estudios consideran que los niveles de $10 \mu \mathrm{M}$ son suficientes para lograr un efecto terapéutico, conclusión lograda en un consenso en el año $2011^{24,25}$.

En resumen, los efectos de la glucosamina traducen el resultado de su actividad antiinflamatoria, la estimulación de la síntesis de proteoglicanos, y la inhibición de las enzimas proteolíticas, lo que disminuye la actividad catabólica de los condrocitos y pudiera tener efecto en la osteoartrosis. La glucosamina también se ha postulado que estimula la producción sinovial de ácido hialurónico e inhibe la actividad de las enzimas lisosomales degradantes de cartílago. Todos estos hallazgos se han demostrado in vitro o en animales, sin la confirmación en humanos ${ }^{26}$.

El condroitín sulfato es un glicosaminoglicano sulfatado, que se encuentra unido a las proteínas y forman un proteoglicano del tipo agrecano, lo que lo hace un importante componente estructural del cartílago que retiene agua y nutrientes, y proporciona resistencia a la compresión y elasticidad; puede ser encontrado en el líquido sinovial y cartílago hialino. Es un GAG que tiene propiedades hidrofílicas, lo que hace que el cartílago absorba grandes cantidades de agua, para incrementar su fuerza compresiva. En estudios clínicos doble ciego a largo plazo, alivia el dolor y aumenta la función de las articulaciones, y de esta manera frena la progresión de la enfermedad ${ }^{27-29}$.

El condroitín sulfato puede ser ingerido, pero no se absorbe de manera rápida desde el tracto gastrointestinal, así, sólo estará biodisponible alrededor del $15 \%$, una vida media de 6 horas y un pico en plasma que oscila entre 2 y 28 horas, a la dosis de $1200 \mathrm{mg}^{29}$.

El condroitín sulfato interactúa con las proteínas en la matriz extracelular y regula una amplia gama de actividades. Su efecto en la OA se cree que es el resultado de la combinación antiinflamatoria y la actividad inmunomoduladora, es decir, la estimulación de la síntesis de proteoglicanos y de ácido hialurónico, y la inhibición de la síntesis de enzimas proteolíticas y del óxido nítrico. Los estudios in vitro han demostrado que la condroitina reduce el factor de necrosis tumoral kappa beta (NF-kB) y la translocación nuclear de la interleuquina IL-1 $\beta$ inducida en los condrocitos. El NF-kB es un factor de transcripción que juega un papel clave en el inicio de diversos genes proinflamatorios implicados en la patogénesis de la OA, de esta manera su reducción podría evitar los cambios en el hueso subcondral; puede reducir la expresión de diversas enzimas proinflamatorias y moléculas como la fosfolipasa A2, la ciclooxigenasa-2, la IL-8, metaloproteasas, proteinasas, y la prostaglandina $\mathrm{E} 2^{26-30}$.

En resumen, tanto el condroitín sulfato como la glucosamina actúan o por la supresión o por la reducción de la expresión de varios mediadores de citoquinas del catabolismo del cartílago. En combinación, se ha demostrado que actúa de manera sinérgica, para modular el metabolismo de la matriz del cartílago articular.

Estos productos han sido recomendados por la European League Against Rheumatism (EULAR) y la European Society for Clinical and Economic Aspects of Osteoporosis and Osteoarthritis (ESCEO). En reciente revisión de la Osteoarthritis Research Society International (OARSI) que la venía recomendando, y ahora basado en opinión de 13 expertos de diversas disciplinas médicas y con evidencia de buena calidad, le dio un porcentaje de riesgo muy bajo y de un moderado a alto tamaño del efecto (hasta 0.75 para el CS) a pesar de ser similar a los DAINEs y superior al paracetamol. La guía inglesa UK National Institute for Health and Clinical Excellence (NICE) no la recomienda por razones económicas. El American College of Rheumatology (ACR) los recomienda solo en determinados casos, porque los datos que soportan su eficacia no están muy claros y no ha sido aprobado por la Food and Drug Administration (FDA), y mientras que para su uso en Europa se necesita receta médica, en USA es un suplemento dietético; pero llama la atención que investigadores norteamericanos recomiendan su uso, sobre todo luego de realizar metaanálisis de 3514 estudios. En América Latina, de manera oficial, solo es recomendada desde el 2010 por la Sociedad Argentina de Reumatología ${ }^{31-39}$.

La OARSI en metaanálisis de 266 trabajos publicados entre enero del 2006 a enero del 2009 llegan a la conclusión de que los resultados son heterogéneos, con muchos errores de sesgo. Las razones, para explicar estos hallazgos, podrían ser: por dosis incorrectas, manufacturas por industrias alimenticias (no farmacéuticas) con compuestos subóptimos, estudios patrocinados por la industria farmacéutica, el origen (la clasificación?) de la OA, la edad de los pacientes, protocolos estandarizados de los puntos a evaluar y la ausencia de un correcto entendimiento de cuándo y cómo aplicarlo, para que puedan aumentar su potencial terapéutico. Incluso se puede atribuir a las bajas concentraciones en los tejidos, por la competencia en el intestino, entre glucosamina y glucosa, para la individualizada captación celular y posterior transporte hacia el hígado y el riñón, que favorece a la glucosa y hace que poca cantidad de glucosamina llegue a la articulación ${ }^{33,40-42}$.

En diferentes estudios en seres humanos se ha informado que disminuye el dolor y mejora la función con 
pocos efectos secundarios, pero existen dos estudios que pretendieron desvirtuar los mencionados efectos, uno el conocido como GAIT (Glucosamine/Chondroitin Arthritis Intervention Trial) ensayo clínico multicéntrico, aleatorio y doble ciego, realizado por el National Institute of Health (NIH) de Estados Unidos, en 1583 pacientes con artrosis de rodilla, que evidenció entre otras posibilidades que la combinación de glucosamina y condroitín sulfato no produjeron reducción del dolor comparado con placebo, y se ha estimado que este hallazgo pudiera ser debido a que el dolor era leve y el paciente no tenía capacidad de distinguir la mejoría con la escala visual, porque, cuando era de moderado a grave lo disminuía de manera significativa con respecto al placebo $(79,2 \%$ vs. $54,3 \%$, $\mathrm{p}=0,002)$ y $10 \%$ superior a la del celecoxib. Es importante resaltar que en este grupo hubo disminución significativa del edema articular $(\mathrm{p}=0,01)^{43-45}$.

El otro informe es el Wandel ${ }^{46}$ que realizó una revisión de 10 trabajos, que incluían 3803 pacientes y concluyó que los productos que contenían glucosamina, condroitín o ambos, no alivian el dolor ni mejoran la pérdida de amplitud de la parte medial de la rodilla, y por tanto no deberían ser usados y recomendaba a las autoridades sanitarias, no tenerlas en su petitorio; a pesar de ello, en su discusión, pone en evidencia que esas investigaciones tenían muchos errores de sesgo. Sobre este metaanálisis, vale la pena señalar la respuesta de Pelletier ${ }^{47}$ que destacaba que los criterios utilizados para la selección de los ensayos no se ajustaban a lo recomendado por la Agencia Europea de Medicamentos (EMA), no consideró estudios de menos de 100 pacientes, no tuvieron en cuenta los valores basales de dolor, el efecto analgésico de la glucosamina/condroitín sulfato no se distribuye de manera uniforme en el tiempo y debido a ser de acción lenta se alcanza de preferencia después de 6 a 12 meses, no incluyó la revisión de más de 5 años de Reginster ${ }^{48,49}$ que demostró la disminución del uso de cirugía con implantes totales, comparado con placebo $(6,3 \% / 14,5 \%)$, no comparó de manera indirecta los trabajos de glucosamina+condroitín sulfato de manera conjunta, con otros productos, y sobre todo que debería haber tomado en cuenta que las metodologías usadas de los diferentes autores, de su metaanálisis, afectan la capacidad de llegar a conclusiones sobre la base de esos resultados. Es de importancia informar que el referido trabajo de Wandel no siguió las pautas de un metaanálisis correcto y la mayoría de respuestas generadas por el mencionado análisis obligó al British Medical Journal a reconocer que las conclusiones negativas del estudio no estaban apoyadas en los datos del mismo estudio y por lo tanto no eran justificadas ${ }^{46-52}$.
Ha sido publicado un ensayo clínico controlado y aleatorizado a dos años, de 605 pacientes, para determinar si de manera efectiva la combinación o de manera individual el sulfato de glucosamina ( $1500 \mathrm{mg}$ diarios) o el condroitín sulfato ( $800 \mathrm{mg}$ diario), comparado con placebo, lograba reducir el "pinzamiento" articular leve y el dolor. La combinación de ambos fármacos, comparados con placebo, resultó en una significativa reducción del pinzamiento $(\mathrm{p}=0,046)$. En cambio, el empleo de cada fármaco en forma individual no obtuvo efecto significativo sobre la estructura articular. Los cuatro grupos redujeron el dolor en el primer año, pero sin diferencias significativas entre ellos. Concluyen que, en este estudio se demuestra que la combinación de GCS y CS, al compararse con placebo, redujo la disminución del espacio tibio-femoral en pacientes con OA que presentan cambios radiológicos "tempranos" 53 .

Lo que se plantea hoy en día es aclarar hechos clínicos referentes a esta combinación terapéutica y su efecto en el envejecimiento del cartílago, pregunta difícil de responder en la actualidad. Existe evidencia que la glucosa, pero no la glucosamina, aceleran el envejecimiento del cartílago. Este hallazgo ha permitido sugerir, que pudiera interferir con el metabolismo de la glucosa a nivel del condrocito, quien expresa en su superficie receptor de insulina, que lo hace sensitivo a sus niveles intra y extracelulares; es decir si los niveles intraarticulares de glucosamina son elevados pueden inhibir la captación de glucosa y proteger esta célula del cartílago ${ }^{54}$.

Los estudiosos de este problema han llegado a la conclusión de que hasta el momento no es un condroprotector, pero existen informes a tres años que previene el deterioro del cartílago, y que pudieran ser considerados modificadores de la enfermedad (DMOADs). De alguna manera protege de la movilidad excesiva o la carga y mejora la marcha, incluso en actividades deportivas, determinadas por biomarcadores, como keratan sulfato, ácido hialurónico, condroitín sulfato y en especial la eliminación urinaria de productos de degradación del telopéptido $\mathrm{C}$ terminal del colágeno (CTX II); pero una vez que cesa la administración del medicamento no se mantiene el efecto. Vale la pena comunicar que existe un informe que puso en evidencia que, en aquellas personas que consumían este producto, había disminución de la mortalidad, de manera especial las portadoras de cáncer y enfermedades respiratorias, a pesar de los errores de sesgo que pudieran existir $^{55-66}$.

En lo relativo a la seguridad a corto y largo plazo es similar al placebo, la mayoría de efectos adversos se relacionan con el aparato gastrointestinal y dado por diarrea, dispepsia y náuseas. Existe controversias relativas al uso 
del medicamento en pacientes diabéticos, y existe un estudio que evidencia que no afecta la glicemia, el metabolismo de la glucosa y la sensibilidad a la insulina, en sujetos sanos, diabéticos o con tolerancia alterada a la glucosa ${ }^{67}$.

En conclusión, la osteoartrosis, en lo que se refiere a sus aspectos diagnósticos como las imágenes y los marcadores bioquímicos, está evolucionando de manera satisfactoria; en lo que se refiere a terapéutica, y en particular el objetivo de esta revisión, que el uso de la glucosamina y el condroitín sulfato en seres humanos es seguro, mejora el dolor y la inflamación, disminuye la utilización de los cada vez menos recomendados, para usarlos de manera continua en el anciano, antiinflamatorios no esteroideos (DAINEs), $\mathrm{y}$ algunos estudios prospectivos, aleatorios, doble ciego y controlados con placebo, demuestran que previenen su evolución y retrasan la posibilidad de una artroplastia de rodilla $^{68}$.

\section{Bibliografía}

1. Guccione AA. Arthritis and the process of disablement. Bibliografia. Phys Ther. 1994 May; 74(5):408-14.

2. Laba TL, Brien JA, Fransen M, Jan S. Patient preferences for adherence to treatment for osteoarthritis: the MEdication Decisions in Osteoarthritis Study (MEDOS). BMC Musculoskelet Disord. 2013 May 6; 14(1):160.

3. Loeser RF: Aging and osteoarthritis. Curr Opin Rheumatol 2011, 23:492-496.

4. Shane Anderson A, Loeser RF: Why is osteoarthritis an age-related disease? Best Pract Res Clin Rheumatol 2010; 24:15-26.

5. Dieppe P. Developments in osteoarthritis. Rheumatology (Oxford). 2011 Feb; 50(2):245-7.

6. Haviv B, Bronak S, Thein R. The complexity of pain around the knee in patients with osteoarthritis. Isr Med Assoc J. 2013 Apr; 15(4):178-81.

7. Neogi T, Bowes MA, Niu J, et al.Magnetic resonance imaging-based three-dimensional bone shape of the knee predicts onset of knee osteoarthritis: data from the osteoarthritis initiative. Arthritis Rheum. 2013 Aug; 65(8):2048-58

8. Mobasheri A. The future of osteoarthritis therapeutics: targeted pharmacological therapy. Curr Rheumatol Rep. 2013 Oct; 15(10):364.

9. Towheed T, Maxwell L, Anastassiades TP, Shea B et al. Glucosamine therapy for treating osteoarthritis. Cochrane Database Syst Rev 2005; (2):CD002946.

10. Pearle A, Warren R, Rodeo S. Basic science of articular cartilage and osteoarthritis. Clin Sports Med $2005 ; 24: 1-12$.

11. Nakamura H, Shibakawa A, Tanaka M, et al. Effects of glucosamine hydrochloride on the production of prostaglandin E2, nitric oxide and metalloproteases by chondrocytes and synoviocytes in osteoarthritis. Clin Exp Rheumatol. 2004; 22(3):293-9.

12. Lin YC, Liang YC, Sheu MT, Lin YC et al. Chondroprotective effects of glucosamine involving the p38 MAPK and Akt signaling pathways. Rheumatol Int. 2008; 28(10):1009-16.

13. Mendis E, Kim MM, Rajapakse N, Kim SK. The inhibitory mechanism of a novel cationic glucosamine derivative against MMP-2 andMMP-9 expressions. Bioorg Med Chem Lett. 2009; 19(10):2755-9.

14. Imagawa K, de Andres MC, Hashimoto K, Pitt D, et al. The epigenetic effect of glucosamine and a nuclear factor-kappa B (NF-kB) inhibitor on primary human chondrocytes-implications for osteoarthritis. Biochem Biophys Res Commun. 2011; 405(3):362-7.

15. Largo R, Alvarez-Soria MA, Díez-Ortego I, Calvo $\mathrm{E}$, et al. Glucosamine inhibits IL-1beta-induced NFkappaB activation in human osteoarthritic chondrocytes. Osteoarthritis Cartilage 2003; 11:290-298.

16. Chan PS, Caron JP. Short-term gene expression changes in cartilage explants stimulated with interleukin beta plus glucosamine and chondroitin sulfate. J Rheumatol 2006; 33:1329-1340.

17. Oegema TRJr, Deloria LB, Sandy JD, Hart DA. Effect of oral glucosamine on cartilage and meniscus in normal and chymopapain-injected knees of young rabbits. Arthritis Rheum 2002; 46:2495-2503.

18. Chan PS, Caron JP. Orth MW. Effect of glucosamine and chondroitin sulfate on regulation of gene expression of proteolytic enzymes and their inhibitors in interleukin-1-challenged bovine Am J Vet Res. 2005 Nov; 66(11):1870-6.

19. Wen $\mathrm{CH}$, Tang CC, Chang YC, Huang SY, et al. Glucosamine sulfate reduces experimental osteoarthritis and nociception in rats: association with changes of mitogen-activated protein kinase in chondrocytes. Wen ZH, Osteoarthritis Cartilage. 2010 Sep; 18(9):1192-202.

20. Naito K, Watari T, Furuhata A, Yomogida S, et al. Evaluation of the effect of glucosamine on an experimental rat osteoarthritis model. Life Sci. 2010 Mar 27; 86(13-14):538-43.

21. Tiraloche G, Girard C, Chouinard L, Sampalis J, et al Effect of oral glucosamine on cartilage degradation in a rabbit model of osteoarthritis.. Arthritis Rheum. 2005 Apr; 52(4):1118-28.

22. Kim MM, Mendis E, Rajapakse N, Kim S-K. Glucosamine sulfate promotes osteoblastic differentiation of MG-63 cells via anti-inflammatory effect. Bioorg Med Chem Lett. 2007; 17(7):1938-42. 
23. Henrotin Y, Mobasheri A, Marty M. Is there any scientific evidence for the use of glucosamine in the management of human osteoarthritis? Arthritis Res Ther. 2012 Jan 30; 14(1):201.

24. Persiani S, Roda E, RovatiL C, Locatelli M, et al.Glucosamine oral bioavailability and plasma pharmacokinetics after increasing doses of crystalline glucosamine sulfate in man. Osteoarthr Cartil. 2005; 6:1041-1049.

25. Henrotin Y, Chevalier X, Herrero-Beaumont G, McAlindon T, et al. Physiological effects of oral glucosamine on joint health: current status and consensus on future research priorities.BMC Res Notes. 2013 Mar 26; 6:115.

26. ShermanAL, Ojeda-Correal G, Mena J, Clinical significance of bone changes in osteoarthritis Ther Adv Musculoskel Dis. 2012; 4(4):259-267.

27. Monfort J, Pelletier J-P, Garcia-Giralt N, Martel-Pelletier J. Biochemical basis of the effect of chondroitin sulfate on osteoarthritis articular tissues. Ann Rheum Dis 2008; 67:735-740.

28. Hochberg MC. Structure-modifying effects of chondroitin sulfate in knee osteoarthritis: an updated meta-analysis of randomized placebo-controlled trials of 2-year duration. Osteoarthritis Cartilage 2010; 18(Suppl 1):S28e31.

29. Miller KL, Clegg DO. Glucosamine and chondroitin sulfate. Rheum Dis Clin North Am. 2011 Feb; 37(1):103-18.

30. Zegels $\mathrm{B}$, Crozes $\mathrm{P}$, Uebelhart $\mathrm{D}$, Bruyère $\mathrm{O}$, Reginster JY. Equivalence of a single dose $(1200 \mathrm{mg})$ compared to a three-time a day dose $(400 \mathrm{mg})$ of chondroitin $4 \& 6$ sulfate in patients with knee osteoarthritis. Results of a randomized double blind placebo controlled study. Osteoarthritis Cartilage. 2013 Jan; 21(1):22-7.

31. Zhang W, Doherty M, Leeb BF, Alekseeva L, et al. EULAR evidence based recommendations for the management of hand osteoarthritis: report of a Task Force of the EULAR Standing Committee for International Clinical Studies Including Therapeutics (ESCISIT). Ann Rheum Dis. 2007; 66(3):377-88.

32. Bruyère $\mathrm{O}$, Cooper $\mathrm{C}$, Pelletier JP, Branco J, et al. An algorithm recommendation for the management of knee osteoarthritis in Europe and internationally: A report from a task force of the European Society for Clinical and Economic Aspects of Osteoporosis and Osteoarthritis (ESCEO). Semin Arthritis Rheum. 2014 May 14. pii: S0049-0172(14)00108-5. doi: 10.1016/j.semarthrit.2014.05.014.

33. Zhang W, Nuki G, Moskowitz RW, et al. OARSI recommendations for the management of hip and knee osteoarthritis: Part III: changes in evidence following systematic cumulative update of research published through January 2009. Osteoarthr Cartil. 2010; 18(4):476-99.

34. McAlindon TE, Bannuru RR, Sullivan MC, Arden $\mathrm{NK}$, et al. OARSI guidelines for the non-surgical management of knee osteoarthritis. Osteoarthritis Cartilage. 2014 Mar; 22(3):363-88.

35. NICE Clinical Guidelines: The care and management of osteoarthritis in adults [www.nice.org.uk/ CG059]

36. Hochberg MC, Altman RD, April KT, Benkhalti $\mathrm{M}$ et al. American College of Rheumatology 2012 recommendations for the use of nonpharmacologic and pharmacologic therapies in osteoarthritis of the hand, hip, and knee. Arthritis Care Res (Hoboken). 2012; 64(4):465-74.

37. Gallagher B, Tjoumakaris FP, Harwood MI, Good $\mathrm{RP}$, et al. Chondroprotection and the Prevention of Osteoarthritis Progression of the Knee: A Systematic Review of Treatment Agents. Am J Sports Med. 2014 May 27. pii: 0363546514533777

38. Martin MS1, Van Sell S, Danter J. Glucosamine and chondroitin: an appropriate adjunct treatment of symptomatic osteoarthritis of the knee. Orthop Nurs. 2012 May-Jun; 31(3):160-6.

39. Primeras guías argentinas de práctica clínica para el diagnóstico y tratamiento de la osteoartritis (OA) de caderas, rodillas y manos. Sociedad Argentina de Reumatología; Edición 2010.

40. Henrotin Y, Marty M, Mobasheric A. What is the current status of chondroitin sulfate and glucosamine for the treatment of knee osteoarthritis? Maturitas. 2014 Jul; 78(3):184-187.

41. Francisco Abad Santos, Dolores Ochoa y Antonio G. García Actualización de la eficacia de condroitín sulfato y sulfato de glucosamina en el tratamiento de la artrosis. Actualidad en Farmacología y Terapéutica junio 2011.Volumen 9(2):97-108.

42. Clegg DO, Reda DJ, Harris CL, Klein MA, et al. Glucosamine, chondroitin sulfate, and he two in combination for painful knee osteoarthritis. N Engl J Med 2006; 354:795-808.

43. National Center for Complimentary and Alternative Medicine. The NIH Glucosamine/Chondroitin Arthritis Intervention Trial (GAIT). J Pain Palliat Care Pharmacother. 2008; 22(1):39-43.

44. Hochberg MC, Clegg DO. Potential effects of chondroitin sulfate on joint swelling: a GAIT report. Osteoarthritis Cartilage 2008; 16 (Supl 3):S22-4.

45. Sawitzke AD, Shi H, Finco MF, Dunlop DD, et al. Clinical efficacy and safety of glucosamine, chondroitin 
sulphate, their combination, celecoxib or placebo taken to treat osteoarthritis of the knee: 2-year results from GAIT. Ann Rheum Dis. 2010 Aug; 69(8):1459-64.

46. Wandel S, Juni P, Tenda 1 B, Nuesch E, et al. Effects of glucosamine, chondroitin, or placebo in patients with osteoarthritis of hip or knee: network meta-analysis. BMJ.2010; 341:c4675.

47. Pelletier JP, Hochberg MC, du Souich P, Kahan A, Michel BA. Glucosamine and osteoarthritis. Effect size is encouraging. BMJ. 2010 Nov 9; 341.

48. Reginster JY, Deroisy R, RovatiL C, Lee RL, Lejeune E, Bruyere O, Giacovelli G, Henrotin Y, Dacre JE, Gossett C. Long-term effects of glucosamine sulphate on osteoarthritis progression: a randomised, placebo-controlled clinical trial. Lancet. 2001 Jan 27; 357(9252):251-6.

49. Reginster JY, Altman RD, Hochberg MC. Prescription glucosamine sulphate is effective in knee osteoarthritis. BMJ 2010; response to 341:c4675

50. Helg A.G. Critical appraisal of the meta-analysis by Wandel et al. - clinical aspects (part 1). http://www.bmj.com/rapid-response/2011/11/03/ critical-appraisal-meta-analysis-wandel-et-al-clinical-aspects-part-1

51. Groves T (2011). Bmj.com.2010.www.bmj.com/content $/ 341 /$ bmj.c4675.

52. Garcia AG. Controversias en el tratamiento de la artrosis con glucosaminoglicanos Editorial. Actualidad en Farmacología y Terapéutica. Junio 2011; volumen 9(2): 85-91.

53. Fransen M, Agaliotis M, Nairn L, Votrubec M, et al. Glucosamine and chond roitin for knee osteoarthritis: a double-blind randomised placebo-controlled clinical trial evaluating single and combination regimens. Ann Rheum Dis. 2014 Jan 6. doi: 10.1136/annrheumdis-2013-203954.

54. Henrotin YE. Interpretation of metaanalyses: pitfalls should be more widely recognized. J Rheumatol. 2012 Jun; 39(6):1107-9.

55. Block JA, Oegema TR, Sandy JD, Plaas A. The effects of oral glucosamine on joint health: is a change in research approach needed? Osteoarthritis Cartilage.2010; 18:5-11.

56. Sodha R, Sivanadarajah N, Alam M. The use of glucosamine for chronic low back pain: a systematic review of randomised control trials. BMJ Open. 2013 Jun 20;3(6).

57. Calamia V, Ruiz-Romero C, Rocha B, et al. Pharmacoproteomic study of the effects of chondroitin and glucosamine sulfate on human articular chondrocytes. Arthritis Res Ther. 2010;6:R138

58. Uitterlinden EJ, Jahr H, Koevoet JL, Bierma-Zeins- tra SM, et al. Glucosamine reduces anabolic as well as catabolic processes in bovine chondrocytes cultured in alginate. Osteoarthritis Cartilage. 2007 Nov; 15(11):1267-7.

59. Davies PS, Graham SM, MacFarlane RJ, et al. Disease-modifying osteoarthritis drugs: in vitro and in vivo data on the development of DMOADs under investigation. Expert Opin Investig Drugs. 2013 Apr; 22(4):423-41.

60. Reginster JY, Deroisy R, Rovati LC, Lee RL et al. Long-term effects of glucosamine sulphate on osteoarthritis progression: a randomised, placebocontrolled clinical trial. Lancet. 2001; 6:251-256.

61. Pavelka K, Gatterova J, Olejarova M, Machacek S et al. Glucosamine sulfate use and delay of progression of knee osteoarthritis: a 3-year, randomized, placebo-controlled, double-blind study. Arch Intern Med. 2002; 6:2113-2123.

62. Ostojic SM, Arsic M, Prodanovic S, Vukovic J et al. Glucosamine administration in athletes: effects on recovery of acute knee injury. Res Sports Med. 2007; 6:113-124.

63. Chen HC, Shah S, Stabler TV, Li YJ et al. Biomarkers associated with clinical phenotypes of hand osteoarthritis in a large multigenerational family: the CARRIAGE family study. Osteoarthr Cartil. 2008; 6:1054-1059.

64. Ohnishi A, Osaki T, Matahira Y, Tsuka T et al. Evaluation of the chondroprotective effects of glucosamine and fish collagen peptide on a rabbit ACLT model using serum biomarkers. J Vet Med Sci. 2013 May 2; 75(4):421-9.

65. Erickson JM, Messer TM. Glucosamine and chondroitin sulfate treatment of hand osteoarthritis. J Hand Surg Am. 2013 Aug; 38(8):1638-40.

66. Bell GA, Kantor ED, Lampe JW, Shen DD et al. Use of Glucosamine and Chondroitin in Relation to Mortality. Eur J Epidemiol. 2012 August; 27(8): 593-603. doi:10.1007/s10654-012-9714-6.

67. Simon RR, Marks V, Leeds AR, Anderson JW. A comprehensive review of oral glucosamine use and effects on glucose metabolism in normal and diabetic individuals. Diabetes Metab Res Rev. 2011; 6:14-27.

68. Bertin P, Taieb C. NSAID-sparing effect of glucosamine hydrochloride in patients with knee osteoarthritis: an analysis of data from a French database. Curr Med Res Opin 2014; 30:271-7. 